\title{
Longitudinal household surveillance for malaria in Rakai, Uganda
}

\author{
Kevin Newell ${ }^{1}$, Valerian Kiggundu ${ }^{2}$, Joseph Ouma ${ }^{3}$, Enos Baghendage ${ }^{4}$, Noah Kiwanuka ${ }^{5}$, Ronald Gray ${ }^{6}$, \\ David Serwadda 3,7 , Charlotte V. Hobbs ${ }^{8}$, Sara A. Healy ${ }^{9}$, Thomas C. Quinn ${ }^{9,10}$ and Steven J. Reynolds 3,9,10,11*
}

\begin{abstract}
Background: HIV and malaria exert co-pathogenic effects. Malaria surveillance data are necessary for public health strategies to reduce the burden of disease in high HIV prevalence settings.

Methods: This was a longitudinal cohort study to assess the burden of malaria in rural Rakai, Uganda. Households were visited monthly for 1 year to identify confirmed clinical malaria (CCM), or parasitaemia with temperature $>37.5^{\circ} \mathrm{C}$, and asymptomatic parasitaemia (AP). Interviews of the adult or child's caregiver and clinical and laboratory assessments were conducted. Rapid diagnostic testing for malaria and anaemia was performed if participants were febrile and anti-malarial treatment given per Uganda Ministry of Health 2010 guidelines. Blood was drawn at every household visit to assess for parasitaemia, and blood smears were assessed at the Rakai Health Science Programme laboratory.
\end{abstract}

Results: A total of 1640 participants were enrolled, including 975 children aged 6 months up to 10 years, 393 adult caregivers, and 272 adolescent/adult household members from 393 randomly selected households in two representative communities. 1459 (89\%) participants completed all study visits. CCM was identified in 304 (19\%) participants, with the highest incidence rate for CCM of 0.38 per person-year (ppy) identified in children $<5$ years, and rates decreased with age; the rates were $0.27,0.16$, and 0.09 ppy for ages $5-<10$ years, $10-<18$ years, and adults $18+$ years, respectively. AP was identified in 943 (57\%) participants; the incidence rate was 1.99 ppy for $<5$ years, 2.72 ppy for $5-<10$ years, 2.55 ppy for $10-<18$ years, and 0.86 ppy among adults, with $92 \%$ of cases being attributed to Plasmodium falciparum by smear. 994 (61 \%) individuals had at least one positive smear; 342 (21\%) had one positive result, 203 (12\%) had two, 115 (7\%) had three, and 334 (21\%) had >3 positive smears during follow-up. Seasonal rates generally followed the rains and peaked during July, then decreased through November before increasing again.

Conclusions: Plasmodium falciparum infection remains high in rural Uganda. Increased malaria control interventions should be prioritized.

Trial registration Clinicaltrials.gov identifier NCT01265407

Keywords: Malaria, Epidemiology, Surveillance, Household, Children, Rakai, Africa

\section{Background}

Malaria is a leading cause of morbidity and mortality in Uganda, accounting for $25-40 \%$ of all outpatient visits at health facilities, $20 \%$ of hospital admissions, and $9-14 \%$ of inpatient deaths [1]. Children under 5 years and

\footnotetext{
*Correspondence: sjr@jhmi.edu

${ }^{11}$ NIAID/NIH ICER Program, c/o US Embassy Kampala, P.O. Box 7007,

Kampala, Uganda

Full list of author information is available at the end of the article
}

pregnant women and HIV-infected individuals bear the greatest burden of disease [2]. Malaria incidence data for this period of time are lacking for most areas of Uganda, particularly in areas of high HIV prevalence, with the most recent Malaria Indicator Surveys having published data collected in 2009 [1] and 2014-2015 [3]. Because HIV and malaria exert co-pathogenic effects [4], malaria surveillance data are necessary for public health strategies to reduce the burden in high HIV prevalence settings. The rural district of Rakai, Uganda, has an HIV prevalence of 
$9 \%$ (age 15-59 years. cf. national prevalence of $7.3 \%$ ) [5]. A study of malaria among children with fever in a hospital setting in the rural Rakai district, Uganda was conducted by this research team and previously described high prevalence of malaria parasitaemia and anaemia among children under 5 years [6]. Because of the continued transmission potential that exists with subclinical parasitaemia, and because many patients do not have ready access to treatment in rural areas, a complementary active-surveillance study was conducted to determine population-based rates of malaria parasitaemia and clinical malaria in Rakai District, and to describe the clinical presentation and prevention strategies being used in households for malaria. This report describes the characteristics of malaria in this rural community with high HIV prevalence [7].

\section{Methods}

Rakai district is on a plateau at an altitude ranging between 750 and $900 \mathrm{~m}$ and has fair rainfall throughout the year, with relatively dry periods during January and February and from June through August. Peak rainfall varies from year to year, but occurs typically in March/ April and October/November [8]. Malaria is meso- to holo-endemic with year round transmission and highest intensity following the rainy seasons or in communities adjacent to lakes and other mosquito breeding sites. Specifically, the Rakai district has high transmission with estimates preceding this study of more than 100 infective bites per person per year [1]. This was a longitudinal household cohort study, with active-surveillance visits by clinicians every 4 weeks during a 1 year follow-up period for each household. The study period spanned from April 2011-September 2012, which included a 6 month recruitment period of randomly selected households from two communities in Rakai District. The communities and households were identified from the Rakai Community Cohort Study (RCCS) described elsewhere [9].

RCCS household enumeration census data were used to identify households with two or more children between the age of 6 months and 10 years. A statistician from RHSP randomly selected eligible households from the census data. A study nurse approached households on each list sequentially to provide information about the surveillance study to the primary caregiver, and then screened the household for eligibility. The study recruited: the primary caregiver of children from each household; all eligible children aged 6 months up to 10 years; and one additional adult or adolescent participant aged 14 years or older. Due to limited resources the study team was unable to recruit every individual in each household; therefore, one additional adult/adolescent participant was randomly selected among eligible adult/ adolescents in the household using the Kish grid method
[10]. This recruitment method employs a pre-assigned table of random numbers to identify a respondent within each household unit with the aim to reduce selection bias.

Study visits were made to enrolled households every 4 weeks during a 1 year surveillance period. Visit procedures included: structured interview of the primary caregiver or legal guardian of the children, clinical and laboratory assessment of each participant in the household, and a heel/finger stick for blood smear; slides were read at a later time at the Rakai Health Sciences Program (RHSP) laboratory. If a participant was febrile $\left(>37.5{ }^{\circ} \mathrm{C}\right)$ the study team also performed a rapid diagnostic test and hemoglobin to assess the need to treat for malaria or anemia. Referrals were made for severe malaria to local clinics for care. Anemia was defined as any hemoglobin level $<11 \mathrm{~g} / \mathrm{dl}$, and further characterized for comparability with the Uganda MIS reports as mild (10-10.9 g/dl), moderate $(8-9.9 \mathrm{~g} / \mathrm{dl})$, or severe $(<8 \mathrm{~g} / \mathrm{dl})$. Data collected during the structured interview included self-reported use of malaria prevention methods, including indoor residual spray, mosquito bed net, insecticide-treated bed net and intermittent preventive treatment.

Confirmed clinical malaria (CCM) was defined as evidence of parasitaemia (positive RDT or smear), accompanied by signs/symptoms consistent with malaria, specifically axillary temperature $>37.5^{\circ} \mathrm{C}$. Treatment for malaria was provided according to the Ugandan Ministry of Health $(\mathrm{MOH})$ guidelines [11] which include artemisinin combination therapy for uncomplicated malaria. Severe malaria was defined in accordance with the World Health Organization (WHO) guidelines [12], with local modifications due to laboratory constraints, as Plasmodium falciparum parasitaemia of any level (or positive RDT) plus one or more of the following: hemoglobin $\leq 5 \mathrm{~g} / \mathrm{dl}$, prostration, respiratory distress, bleeding, massive haemoglobinuria, recent seizures, coma or obtundation, inability to eat or drink, persistent vomiting or jaundice. Participants identified with suspected severe malaria were referred immediately for in-patient treatment at the nearest health center or hospital.

\section{Statistical methods}

This study is a descriptive, non-comparative study to assess the epidemiology of malaria infection in children aged 6 months $-<10$ years, and adults living in same households as children. The main objective is to estimate the incidence of asymptomatic and symptomatic malaria in the study population in preparation for future malaria studies in Rakai, including malaria vaccine trials. As data was lacking on malaria incidence in Rakai communities, and on the variance in children aged 6 months $-<10$ years, sample size calculations were based 
on a method [13] that applies a pre-determined ratio between the precision width and the standard deviation to estimate sample sizes for descriptive studies using the formula $\mathrm{N}=4 \mathrm{z}^{2} \mathrm{~S}^{2} / \mathrm{W}^{2}$. A precision width that is $10 \%$ of the standard deviation is narrow enough to provide a large enough sample size that would adequately describe the incidence of malaria in the study population. Using a $95 \%$ confidence interval (CI) and a ratio of interval width to standard deviation (W/S) of 0.10 requires a total sample size of 1537 participants. A low loss to follow-up rate of $3 \%$ was assumed and by adjusting for a non-response rate of $3 \%$, the required total sample size was determined to be 1635 [1537/(1-0.06)] participants. The unit of enrolment was the household; therefore, an adequate number of households were enumerated from RCCS census data using known household age cohort structure within these Rakai communities in order to approximate this sample size. Malaria prevalence is reported as proportion of subjects identified during the study period with $\mathrm{CCM}$ or AP and stratified by subject characteristics, including age, sex and community. A Chi square statistic was used to compare prevalence across age and gender strata. Incidence estimates are reported per person-year with $95 \%$ CI. A kappa statistic was calculated to measure the level of agreement between RDT and blood smear findings. The malaria attributable fraction (MAF) among febrile subjects is also reported along with $95 \% \mathrm{CI}$ for these estimates to provide a measure of the proportion of fevers identified during this study period which can be attributed to malaria. All analyses were conducted using SAS software version 9.4. Copyright, SAS Institute Inc. SAS and all other SAS Institute Inc. product or service names are registered trademarks or trademarks of SAS Institute Inc., Cary, NC, USA.

\section{Laboratory procedures}

Laboratory evaluations included the following at all scheduled surveillance visits: Hemocue ${ }^{\circledR}$ rapid haemoglobin test for febrile subjects and malaria rapid diagnostic test (Malaria Total Quick Test, Cypress Diagnostics, Langdorp, Belgium, sensitivity $99.7 \%$ and specificity $99.5 \%$.) for febrile subjects; thick and thin slide malaria smears were obtained from all subjects by finger- or heelprick and evaluated in the RHSP laboratory. The malaria “Total Quick" RDT tests for both antigens specific to $P$. falciparum (HRP2) as well as lactate dehydrogenase, which is an antigen shared by other Plasmodium species that infect humans, including P. falciparum, Plasmodium ovale, Plasmodium malariae, and Plasmodium vivax. Thick smears were used to identify parasites and quantify parasite count. Thin smears were used for parasite speciation. All slides were read in the RHSP Lab by experienced microscopists who were blinded to the history or signs of clinical malaria. Internal quality assurance of smear reads was conducted by preserving slides and having a separate blinded microscopist reread $10 \%$ of all slides with any discrepant resolved by a third microscopist.

\section{Ethics}

The study was approved by the Uganda Virus Research Institute Science and Ethics Committee, the Uganda National Council for Science and Technology, and the National Institute of Allergy and Infectious Diseases Intramural Institutional Review Board. All study participants at least 18 years of age provided written informed consent prior to screening. Parents or guardians of minors provided written consent and children aged 8 years and older provided assent to the research.

\section{Results}

The primary caregivers in 403 households were visited in their homes and provided information about the study, of which 393 (98\%) agreed to participate. Of the ten households not enrolled, four caregivers refused participation, three caregivers were unable to be contacted, two households had all children away at boarding school, and one household had migrated out of the study area. A total of 1640 participants were enrolled, including 393 $(24 \%)$ adult caregivers, $975(59 \%)$ children $<10$ years of age, and $272(17 \%)$ other adult or adolescent members of the households. An average of 2.5 child participants, and an average of 4.2 total participants, were enrolled per household. Table 1 presents a summary of enrolment characteristics.

Retention in the annual surveillance study was high with 1459 (89\%) of participants completing the final study visit. Visit adherence was also very high, with only 481 (2.2\%) of 21,618 expected study visits missed; 21,137 (97.8\%) participant visits were completed during the surveillance period. 108 (6.6\%) participants were lost to follow-up, twenty-two (1.3\%) participants withdrew consent, and there were four $(0.2 \%)$ deaths. Forty-seven $(2.9 \%)$ of participants were terminated due to other circumstances.

There were 21,128 blood smears obtained during the study period. CCM was identified in 304 (19\%) participants (Table 2), with highest proportion of CCM in children $<5$ years (33\% for males; $25 \%$ for females, $\mathrm{p}<0.0001)$. CCM prevalence decreased with age, with the lowest rate identified among adult females (7\%). The rate of CCM was consistently higher among males than females in all age categories $(\mathrm{p}<0.0001)$. AP was identified in 943 (57\%) participants during the study period. The prevalence of AP was highest in older children $(5-<10$ years $)$ and adolescents $(10-<18$ years $)$ and lowest in children under five and adults (18+ years). The 
Table 1 Enrollment by community, cohort and gender

\begin{tabular}{|c|c|c|c|c|c|}
\hline & \multicolumn{2}{|c|}{ Kalisizo n (\%) } & \multicolumn{2}{|c|}{ Kabira n (\%) } & \multirow[t]{2}{*}{ Total n (\%) } \\
\hline & Male & Female & Male & Female & \\
\hline \multicolumn{6}{|l|}{ Cohort } \\
\hline Caregivers & $07(2)$ & $202(51)$ & $05(1)$ & $179(46)$ & $393(24)$ \\
\hline $\begin{array}{l}\text { Children } \\
\quad<10 \text { years }\end{array}$ & $292(30)$ & $231(24)$ & $235(24)$ & $217(22)$ & $975(59)$ \\
\hline $\begin{array}{l}\text { Additional adult/ } \\
\text { adolescents }\end{array}$ & $77(28)$ & $62(23)$ & $66(24)$ & $67(25)$ & $272(17)$ \\
\hline Total & $376(23)$ & $495(30)$ & 306 (19) & 463 (28) & 1640 \\
\hline
\end{tabular}

prevalence of AP did not differ by gender among children $<10$ years; however, in older children, adolescents and adults, males had $\sim 10 \%$ higher proportion of AP within each age stratum.
Incidence rate estimates for malaria are presented according to age and gender strata (Table 3). Overall incidence of CCM in this study was 0.23 per person-year (ppy). Rates of CCM follow the same general trend as for prevalence, with youngest children $<5$ years bearing the greatest burden of malaria (0.38 ppy), and decreasing rates of malaria with increasing age $(0.27$ ppy in children $5-<10$ years, 0.16 ppy in ages $10-<18$ years, and 0.09 ppy in adults $18+$ ). Incidence of AP was 1.99 ppy overall, and remained high across all age cohorts: the rate estimate is 1.99 ppy in young children $<5$ years, 2.72 in children $5-<10$ years, 2.55 ppy in ages $10-<18$ years, and 0.86 ppy in adults $18+$.

Figure 1 shows the frequency of positive smear results (any level of parasitaemia) during the study period. 646 (39\%) had no positive smears; 994 (61 \%) individuals had at least one positive smear of which $342(21 \%)$ had one positive result, 203 (12\%) had two, 115 (7\%) had three,

Table 2 Prevalence of confirmed clinical malaria and asymptomatic parasitaemia by age and gender

\begin{tabular}{|c|c|c|c|c|c|c|c|}
\hline \multirow[t]{2}{*}{ Age (years) } & \multicolumn{2}{|c|}{ Confirmed clinical malaria n (\%) } & \multicolumn{2}{|c|}{ Asymptomatic parasitaemia $\mathrm{n}(\%)$} & \multicolumn{3}{|c|}{ Total participants n (\%) } \\
\hline & Male & Female & Male & Female & Male & Female & Total \\
\hline \multirow[t]{2}{*}{$0.5-<5$} & $76(33)$ & $45(25)$ & $128(56)$ & $104(58)$ & $229(56)$ & $179(44)$ & $408(25)$ \\
\hline & $121(30)$ & & $232(57)$ & & & & \\
\hline \multirow[t]{2}{*}{$05-<10$} & $68(23)$ & $52(19)$ & $196(66)$ & $169(63)$ & $298(53)$ & $269(47)$ & $567(35)$ \\
\hline & $120(21)$ & & $365(64)$ & & & & \\
\hline \multirow[t]{2}{*}{$10-<18$} & $13(13)$ & $15(14)$ & $71(72)$ & $68(61)$ & $99(47)$ & $111(53)$ & $210(13)$ \\
\hline & $28(13)$ & & $139(66)$ & & & & \\
\hline \multirow[t]{2}{*}{$18+$} & $7(12)$ & $28(7)$ & $31(55)$ & $176(44)$ & $56(12)$ & $399(88)$ & $455(28)$ \\
\hline & $35(8)$ & & $207(45)$ & & & & \\
\hline \multirow[t]{2}{*}{ Total } & $164(24)$ & $140(15)$ & $426(62)$ & $517(54)$ & $682(42)$ & $958(58)$ & 1640 \\
\hline & $304(19)$ & & $943(57)$ & & & & \\
\hline
\end{tabular}

Table 3 Incidence rate (per person-year) of confirmed clinical malaria and asymptomatic parasitaemia by age and gender

\begin{tabular}{|c|c|c|c|c|}
\hline \multirow[t]{2}{*}{ Age (years) } & \multicolumn{2}{|c|}{ Confirmed clinical malaria $(95 \% \mathrm{Cl})$} & \multicolumn{2}{|c|}{ Asymptomatic parasitaemia $(95 \% \mathrm{Cl})$} \\
\hline & Male & Female & Male & Female \\
\hline \multirow[t]{2}{*}{$0.5-<5$} & $0.43(0.34,0.51)$ & $0.32(0.23,0.40)$ & $1.97(1.64,2.29)$ & $2.01(1.58,2.44)$ \\
\hline & $0.38(0.32,0.44)$ & & $1.99(1.72,2.25)$ & \\
\hline \multirow[t]{2}{*}{$05-<10$} & $0.29(0.23,0.35)$ & $0.26(0.19,0.33)$ & $2.86(2.55,3.17)$ & $2.56(2.21,2.91)$ \\
\hline & $0.27(0.23,0.32)$ & & $2.72(2.47,2.97)$ & \\
\hline \multirow[t]{2}{*}{$10-<18$} & $0.19(0.11,0.27)$ & $0.13(0.08,0.19)$ & $2.70(2.19,3.20)$ & $2.43(1.95,2.90)$ \\
\hline & $0.16(0.12,0.21)$ & & $2.55(2.20,2.90)$ & \\
\hline \multirow[t]{2}{*}{$18+$} & $0.17(0.07,0.28)$ & $0.08(0.05,0.11)$ & $1.22(0.92,1.52)$ & $0.81(0.70,0.93)$ \\
\hline & $0.09(0.06,0.12)$ & & $0.86(0.75,0.96)$ & \\
\hline \multirow[t]{2}{*}{ Total } & $0.31(0.27,0.35)$ & $0.18(0.15,0.21)$ & $2.40(2.19,2.61)$ & $1.70(1.54,1.85)$ \\
\hline & $0.23(0.21,0.26)$ & & $1.99(1.86,2.11)$ & \\
\hline
\end{tabular}




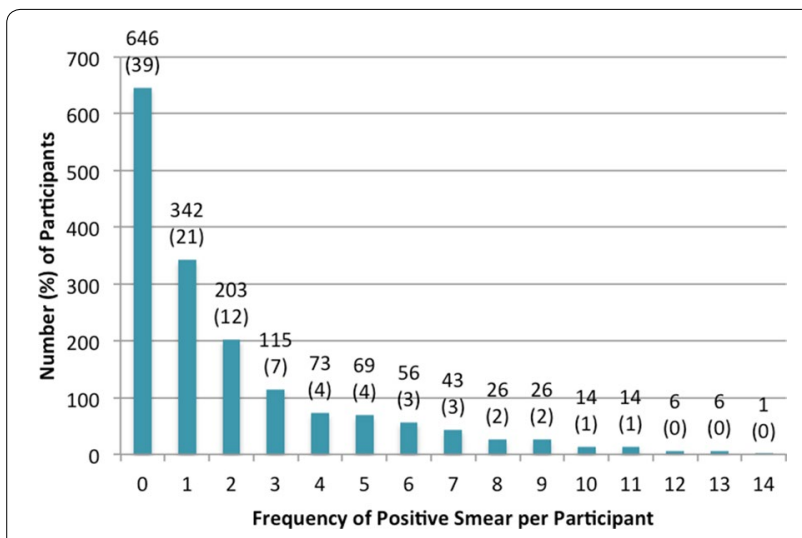

Fig. 1 Frequency of positive smears for each participant during follow-up data value for each bar is number (\%) of participants

and $334(21 \%)$ had $>3$ positive smear results during follow-up.

Figure 2 shows monthly prevalence of smear positive malaria outcomes during the study period. Seasonal rates generally followed the rains [8] and peaked during April to July, then decreased through November before increasing again.

There were speciation results for 3267 positive smear readings. Plasmodium falciparum was identified in $92 \%$, $P$. vivax in $6 \%$, and P. malariae in $6 \%$ of smears. The predominance of $P$. falciparum was parallel in both clinical malaria and asymptomatic parasitemia groups (Table 4).
There were 113 instances of multi-species infection, with 72 (2 \%) cases identified with both $P$. falciparum and $P$. vivax; 40 (1\%) cases, with both $P$. falciparum and $P$. malariae; and one $(0.03 \%)$ case with both $P$. vivax and $P$. malariae.

Fever (temperature of $>37.5{ }^{\circ} \mathrm{C}$ ) was identified at 467 (2.2\%) visits. 468 rapid diagnostic tests for malaria were performed in febrile participants and 347 (74.2\%) RDT samples were positive. Subsequent confirmation of these results by thick smear showed 242 (52\%) smear-positivity. There were concordant positive results for 218 (47 \%) samples, concordant negative results for 113 (24\%) samples, and discordant results for 137 (29 \%) samples: eight (2\%) samples were RDT-negative but smear-positive, and 129 (28\%) samples were RDT-positive, but smear-negative. The kappa statistic for level of agreement between RDT and smear result was 0.42 (95\% C.I. 0.35, 0.49).

The malaria attributable fraction among febrile episodes was consistent across age groups: $67 \%$ among young children $<5$ years, $68 \%$ in children $5-<10$ years, $66 \%$ in ages $10-<18$ years, and $70 \%$ in adults $18+$ years. There was a notable difference between genders that was consistent across all age groups, with males demonstrating a higher fraction of fevers attributable to malaria. The MAF for fever among male participants was $76 \%$, whereas for females it was $58 \%$.

Prevalence of anaemia was determined for all febrile participants and results are presented in Table 5 only

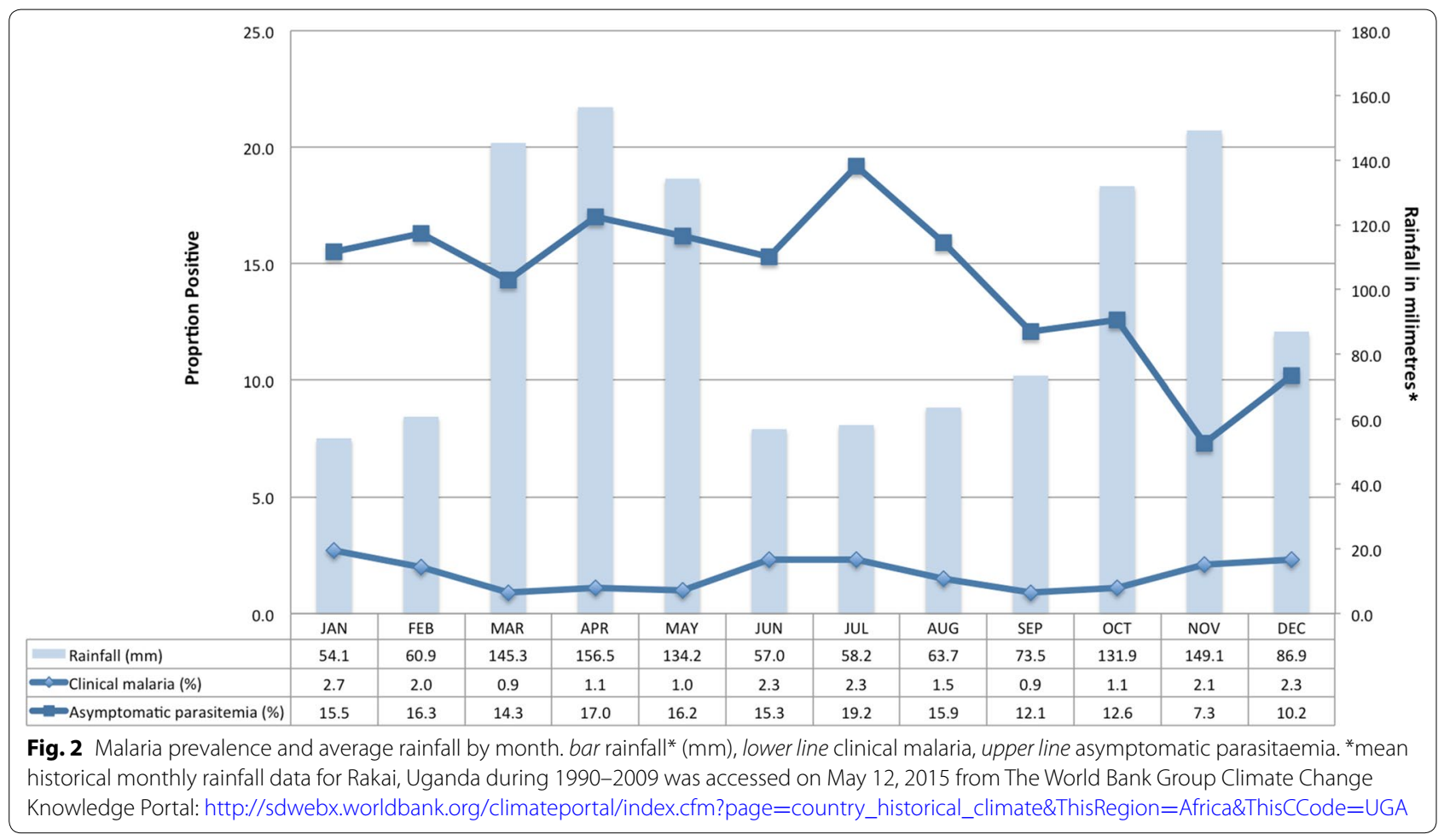


Table 4 Speciation observed for positive smears $(\mathrm{N}=3267)$

\begin{tabular}{lccc}
\hline Malaria species & $\begin{array}{l}\text { Confirmed clinical } \\
\text { malaria } \mathbf{n}(\%)\end{array}$ & $\begin{array}{l}\text { Asymptomatic } \\
\text { parasitaemia } \mathbf{n}(\%)\end{array}$ & Total $\mathbf{n}(\%)$ \\
\hline Plasmodium falciparum & $220(7)$ & $2778(93)$ & $2998(92)$ \\
Plasmodium malariae & $3(2)$ & $193(98)$ & $196(6)$ \\
Plasmodium vivax & $10(5)$ & $176(95)$ & $186(6)$ \\
\hline
\end{tabular}

Multiple species were identified for 113 positive smears, so the total count shows as higher than the number of positive slides

Table 5 Prevalence of anemia among children age 6 months $-<10$ years

\begin{tabular}{|c|c|c|c|c|c|}
\hline & $\begin{array}{l}\text { Severe anemia }(<8 \mathrm{~g} / \mathrm{dl}) \\
\mathrm{n}(\%)\end{array}$ & $\begin{array}{l}\text { Moderate anemia } \\
(8-9.9 \mathrm{~g} / \mathrm{dl}) \mathrm{n}(\%)\end{array}$ & $\begin{array}{l}\text { Mild anemia } \\
(10-10.9 \mathrm{~g} / \mathrm{dl}) \mathrm{n}(\%)\end{array}$ & $\begin{array}{l}\text { Normal hemoglobin } \\
(11+\mathrm{g} / \mathrm{dl}) \mathrm{n}(\%)\end{array}$ & Count $(\mathrm{N}=336)$ \\
\hline \multicolumn{6}{|c|}{ Age group (years) } \\
\hline $0.5-<5$ & $15(8)$ & $26(15)$ & $41(23)$ & $95(54)$ & $177(53)$ \\
\hline $05-<10$ & $5(3)$ & $21(13)$ & $14(9)$ & $119(75)$ & $159(47)$ \\
\hline \multicolumn{6}{|l|}{ Gender } \\
\hline Male & $14(7)$ & $25(13)$ & $30(16)$ & $119(63)$ & $188(56)$ \\
\hline Female & $6(4)$ & $22(15)$ & $25(17)$ & $95(64)$ & $148(44)$ \\
\hline \multicolumn{6}{|l|}{ Cluster } \\
\hline Kalisizo & $7(4)$ & $29(17)$ & $31(18)$ & $103(61)$ & $170(51)$ \\
\hline Kabira & $13(8)$ & $18(11)$ & $24(14)$ & $111(67)$ & $166(49)$ \\
\hline \multicolumn{6}{|c|}{ Confirmed clinical malaria status } \\
\hline Yes & $20(8)$ & $41(16)$ & $52(20)$ & $144(56)$ & $257(76)$ \\
\hline No & $0(0)$ & $6(8)$ & $3(4)$ & $70(89)$ & $79(24)$ \\
\hline Total & $20(6)$ & $47(14)$ & $55(16)$ & $214(64)$ & 336 \\
\hline Any anaemia & $122(36)$ & & & & \\
\hline
\end{tabular}

for participants $<10$ years since anaemia was very rare in older age groups. Among 336 children $<10$ with fever, $122(36 \%)$ showed anaemia of any severity level, of which 113 (93 \%) had CCM. All 20 children identified with severe anaemia (hemoglobin $<8 \mathrm{~g} / \mathrm{dl}$ ) were CCM cases, and CCM cases were 3.86 times more likely to be anaemic compared to children without CCM ( $p<0.0001)$. Younger children $<5$ years were 1.84 times more likely to be anaemic compared to older children $5-<10$ years ( $\mathrm{p}<0.0001)$.

Self-reported (or caregiver-reported for children) prevention methods used at baseline are summarized in Table 6. 192 (12\%) participants reported use of indoor residual spray (IRS) in the household, 656 (40 \%) reported use of any mosquito bed net, of which 473 (29\%) reported the bed net was an insecticide-treated net (ITN), and 31 ( $2 \%$ ) reported using intermittent preventive treatment (IPT) for malaria. Caregivers reported using bed nets more frequently than other cohorts $(\mathrm{p}<0.001)$.

\section{Discussion}

This study describes the relatively high rates of malaria in rural Uganda that also has a high burden of HIV [7].
This is compatible with other studies in rural Uganda. A strength of this study is the longitudinal monthly assessments at the household level among a large number of individuals over a 1 year period and across a spectrum of age groups. Loss to follow-up was minimal and adherence was high. The study provides estimates of malaria incidence rather than prevalence alone, and during a time frame in rural Uganda not described by the periodic MIS rounds. Furthermore, the study also provides information on malaria prevention methods, and reports on prevalence of anaemia among febrile children. There are some important limitations of this study. Recruitment targeted households from two community clusters within Rakai district that provided convenient access to the study team due to their proximity to health centers and therefore these findings might not be generalizable to the entire district. The investigators did not ascertain HIV sero-status as part of the study; however, we these results are interpretable within the context of an area of known endemic HIV transmission and prevalence.

Findings from this study are consistent with reports from the Uganda MIS from 2009 to 2014/2015 (Rakai District is included in the Central 1 region), which show a downward trend for prevalence of malaria and anaemia 
Table 6 Self-reported malaria prevention methods at baseline

\begin{tabular}{llllll}
\hline & $\begin{array}{l}\text { Use of indoor } \\
\text { residual spray } \mathbf{n}(\%)\end{array}$ & $\begin{array}{l}\text { Use of any mosquito } \\
\text { net } \mathbf{n}(\%)\end{array}$ & $\begin{array}{l}\text { Use of insecticide- } \\
\text { treated } \mathbf{n e t} \mathbf{n}(\%)\end{array}$ & $\begin{array}{l}\text { Use of intermittent pre- } \\
\text { ventive treatment } \mathbf{n}(\%)\end{array}$ & $\begin{array}{c}\text { Count (N=1640) } \\
\text { Cohort }\end{array}$ \\
Caregiver & $46(12)$ & & & & \\
Adult & $27(10)$ & $197(50)$ & $137(35)$ & $28(7)$ & $393(24)$ \\
Child & $119(12)$ & $80(29)$ & $59(22)$ & $1(0.7)$ & $272(17)$ \\
Total & $192(12)$ & $379(39)$ & $277(28)$ & $31(2)$ & $975(59)$ \\
\hline
\end{tabular}

in young children age 0-59 months. The current study from $2011 / 2012$ is in between these MIS time points, and prevalence estimates for CCM in the youngest age cohort likewise fall in between (30\% in this study, versus $45 \%$ from the MIS in 2009 and $13 \%$ in 2014/2015). Prevalence of anaemia follows a similar trend, with $8 \%$ of the youngest age cohort from the current study identified with severe anaemia (hemoglobin $<8 \mathrm{~g} / \mathrm{dl}$ ), compared to 11 and $4 \%$ respectively from the MIS 2009 and 2014/2015; and $46 \%$ of children $<5$ years from this study identified with any level of anaemia (hemoglobin $<11 \mathrm{~g} /$ dl) compared to $63 \%$ form the MIS 2009. Reported use of malaria prevention strategies in the current study is also comparable to the Uganda MIS findings. Overall use of any mosquito bed net (40 \%) and ITN (29\%) fall in between an apparent increasing trend reported in MIS (any bed net use in children was $32 \%$ in 2009 and $68 \%$ in $2014 / 2015$, with use of ITN $22 \%$ for children $<5$ years in 2009 , and $59 \%$ in 2014/2015).

As expected, the predominant species identified by microscopy was $P$. falciparum. The identification of $P$. vivax in East Africa is not unexpected, although this identification was done by smear and not by PCR. The presence of $P$. vivax in Duffy-negative individuals, as would be expected in East Africa, has been previously described in East Africa [14] and in the Amazon [15]. It is also possible that the specificity of microscopy was not optimal.

The discrepancy between RDT and smear positivity is also not unexpected but is higher than has been observed in other studies [1,3]: for the $2 \%$ of samples that were RDT negative but smear positive, it is possible that nonfalciparum species accounted for the RDT "false negatives" since the sensitivity for non-falciparum species with this RDT is slightly lower. Indeed, for those eight (2\%) discordant samples of the samples we reported on, six $(75 \%)$ were positive for P. falciparum, one (12\%) was positive for $P$. vivax, and one $(12 \%)$ was positive for $P$. malariae. For the $28 \%$ of RDTs that were positive but smear negative, it is possible that microscopy sensitivity was imperfect, or that the RDTs were picking up circulating antigen or parasites (gametocytes) that existed after patients had been recently treated for infection [16].
A higher degree of AP than CCM was observed in this study. If molecular methods had been employed in this study, we likely would have detected higher AP rates. Indeed, using sensitive molecular methods and targeting interventions to those with AP is important to eradication since it likely fuels the cycle of transmission. Community-wide drug treatment, indoor residual spraying, bed net distribution, and mosquito larviciding are all efforts that have been and could be guided by highly sensitive community screening and intervention efforts [17].

It is likely that children who had febrile illnesses who were RDT negative had infections other than malaria, and this has been increasingly described in the literature recently. In fact, recent studies have shown that with the decreasing incidence of malaria as evidenced by data in this study and the Uganda MIS 2009 and $2014 / 2015$ [1,3], etiologies of fever in younger children are much more likely to be due to viral illnesses than bacterial or parasitic infections (such as typhoid or malaria) [18].

\section{Conclusions}

Plasmodium falciparum infection remains high in rural Rakai, Uganda. Increased malaria interventions should be implemented to reduce the burden of disease.

\section{Authors' contributions}

SJR, DS, RG, NK and TCQ designed the study and provided administrative, clinical and laboratory oversight. VK, JO and EB were responsible for the daily management of the research study and data collection process. KN was responsible for data quality management, conducted the statistical analyses, and drafted the manuscript. CVH and SAH provided expert advice on data analysis and manuscript writing. All authors contributed to the manuscript. All authors read and approved the final manuscript.

\section{Author details}

${ }^{1}$ Research Data and Communication Technologies, Inc., Garrett Park, MD, USA. ${ }^{2}$ Office of HIV/AIDS/Global Health Bureau, USAID Global Health Fellows Program, 1300 Pennsylvania Avenue NW, Washington 20523, DC, USA. ${ }^{3}$ Rakai Health Sciences Program, Kalisizo, Uganda. ${ }^{4}$ Makerere University Walter Reed Project, Kampala, Uganda. ${ }^{5}$ International AIDS Vaccine Initiative (IAVI), Uganda Program, Entebbe, Uganda. ${ }^{6}$ Johns Hopkins Bloomberg School of Public Health, Baltimore, MD, USA. ${ }^{7}$ School of Public Health, Makerere College of Health Sciences, Kampala, Uganda. ${ }^{8}$ Batson Children's Hospital, Division of Infectious Disease, Department of Pediatrics, University of Mississippi Medical Center, Jackson, MS, USA. ${ }^{9}$ Division of Intramural Research, National Institute of Allergy and Infectious Diseases, National Institutes of Health, Bethesda, MD, USA. ${ }^{10}$ Johns Hopkins University School of Medicine, Baltimore, 
MD, USA. ${ }^{11}$ NIAID/NIH ICER Program, c/o US Embassy Kampala, P.O. Box 7007, Kampala, Uganda.

\section{Acknowledgements}

This project has been funded in part from the National Cancer Institute, National Institutes of Health, under Contract No. HHSN261200800001E and in part by the Division of Intramural Research of the National Institute of Allergy and Infectious Diseases. The content of this publication does not necessarily reflect the views or policies of the Department of Health and Human Services, nor does mention of trade names, commercial products, or organizations imply endorsement by the U.S. Government. The content of this manuscript is the sole responsibility of the authors. The information provided here is not official U.S. Government information and does not necessarily represent the views or positions of United States Agency for International Development, the United States Department of State, nor the United States Government. We are most grateful to the study participants and the Rakai Community Advisory Board whose commitment and cooperation made this study possible.

\section{Competing interests}

The authors declare that they have no competing interests.

Received: 13 May 2015 Accepted: 25 January 2016

Published online: 09 February 2016

\section{References}

1. Uganda Bureau of Statistics (UBOS) and ICF Macro. Uganda Malaria Indicator Survey 2009. Calverton: UBOS and ICF Macro; 2010.

2. Uganda Ministry of Health. Uganda Malaria programme review report 2001-2010. Uganda: Kampala; 2011.

3. Uganda Bureau of Statistics (UBOS) and ICF International. 2015. Uganda Malaria Indicator Survey 2014-15: Key Indicators. Kampala, and Rockville: UBOS and ICF International.

4. Malamba S, Hladik W, Reingold A, Banage F, McFarland W, Rutherford G, et al. The effect of HIV on morbidity and mortality in children with severe malarial anaemia. Malar J. 2007;6:143.
5. Uganda Ministry of Health. Uganda AIDS Indicator Survey 2011. Kampala, Uganda.

6. Kiggundu VL, O'Meara WP, Musoke R, Nalugoda FK, Kigozi G, Baghendaghe $\mathrm{E}$, et al. High prevalence of malaria parasitemia and anemia among hospitalized children in Rakai, Uganda. PLoS One. 2013;8:e82455.

7. Grabowski MK, Lessler J, Redd AD, Kagaayi J, Laeyendecker O, Ndyanabo A, et al. The role of viral introductions in sustaining community-based HIV epidemics in rural Uganda: evidence from spatial clustering, phylogenetics, and egocentric transmission models. PLoS Med. 2014;1 1:e1001610.

8. Rakai District Local Government, Production and Marketing Department. Three-Year Production Sector Development Plan. Planning Period: 2010/2011-2012/2013.

9. Wawer MJ, Sewankambo N, Serwadda D, Quinn TC, Paxton LA, Kiwanuka $\mathrm{N}$, et al. Control of sexually transmitted diseases for AIDS prevention in Uganda: a randomized community trial. Lancet. 1999;353:525-35.

10. Kish L. A procedure for objective respondent selection within the household. J Am Stat Assoc. 1949;44:380-7.

11. Uganda Ministry of Health. Clinical Treatment Guidelines 2010, Kampala, Uganda.

12. WHO, Guidelines for the Treatment of Malaria. Geneva: World Health Organization, 2010.

13. Hulley S, Cummings SR, Browner WS, Grady D, Hearst N, Newman TB. Designing clinical research. 2nd ed. Lippincott: Williams \& Wilkins; 2001.

14. Ryan JR, Stoute JA, Amon J, Dunton RF, Mtalib R, Koros J, et al. Evidence for transmission of Plasmodium vivax among a Duffy antigen negative population in Western Kenya. Am J Trop Med Hyg. 2006;75:575-81.

15. Pasvol G. Eroding the resistance of Duffy negativity to invasion by Plasmodium vivax? Trans R Soc Trop Med Hyg. 2007;101:953-4.

16. Murphy SC, Shott JP, Parikh S, Etter P, Prescott WR, Stewart VA. Malaria diagnostics in clinical trials. Am J Trop Med Hyg. 2013;89:824-39.

17. Bousema T, Okell L, Felger I, Drakeley C. Asymptomatic malaria infections: detectability, transmissibility and public health relevance. Nat Rev Microbiol. 2014;12:833-40.

18. D’Acremont V, Kilowoko M, Kyungu E, Philipina Sister, Sangu W, KahamaMaro J, et al. Beyond malaria-causes of fever in outpatient Tanzanian children. N Engl J Med. 2014;370:809-17.

\section{Submit your next manuscript to BioMed Central and we will help you at every step:}

- We accept pre-submission inquiries

- Our selector tool helps you to find the most relevant journal

- We provide round the clock customer support

- Convenient online submission

- Thorough peer review

- Inclusion in PubMed and all major indexing services

- Maximum visibility for your research

Submit your manuscript at www.biomedcentral.com/submit 